\title{
Bilateral absence of vas deferens (BAVD) a case report
}

\begin{abstract}
Mutations in transmembrane conductance of cystic fibrosis (CFTR) are found in men in couples followed for infertility and for which azoospermia associated with congenital bilateral absence of the deferential canal (CBAVD) were found in men. There is a frequent association between CFTR and CBAVD abnormalities. CBAVD exists in almost all men with cystic fibrosis and causes an obstructive azoospermia that cannot be treated surgically; this poses a diagnostic and therapeutic problem because its management remains complex. However, with the use of assisted reproductive techniques (ART), in particular the aspiration of testicular or epididymic sperm, the injection of intracytoplasmic sperm and in vitro fertilization, it is possible that men with CBAVD can produce offspring. We report the case of a 32-year-old patient who consulted for primary infertility evolving over the past 5 years (genetic advice was provided). The aim of this article is to show the diagnostic and therapeutic difficulties related to this particular form of male primary infertility. Recent assisted human reproduction techniques like Intracytoplasmic Sperm Injection (ICSI) or In Vitro Fertilization (FIV) offer very good results for couple consulting for infertility of man with CBAVD.
\end{abstract}

Key note message: Surgical sperm collection is the hope for obtaining a pregnancy from infertility couples consulting for azoospermia associated with congenital bilateral absence of the vas deferens (CBAVD).

Keywords: azoospermia, vas deferens, congenital bilateral absence of the vas deferens, genetic counselling, infertility, cystic fibrosis
Volume II Issue 3 - 202 I

\section{Mzyiene Mohammed, Ziba Ouima Justin Dieudonné, Adil Mellouki, Mustapha Ahsaini, Mellas Soufiane, Mohammed Fadl Tazi, Jalal Eddine Elammari, Mohammed Jamal Elfassi, Moulay Hassan Farih}

Department of Urology, Centre Hospitalier Universitaire Hassan 2, University Sidi Mohamed Ben Abdellah, Morocco

Correspondence: Mzyiene Mohammed, Ziba OJD, Department of Urology, Centre Hospitalier Universitaire Hassan, University Sidi Mohamed Ben Abdellah, Morocco, Email dr.mzyiene@gmail.com, ouimzib@gmail.com

Received: April 29, 202I | Published: May 14, 2021

\section{Introduction}

One of the aetiologies' of the obstructive azoospermia is related to an anomaly of the vas deferens and seminal vesicles. This pathology has become a public health problem among infertile couples. ${ }^{1}$ Mutations of cystic fibrosis (CFTR) are the predominant aetiology of CBAVD.

Clinically, the symptomatology of congenital absence of vas deferens is based on the appearance of the testicles, the absence of seminal vesicles and a hormonal anomaly. ${ }^{2}$ The analysis of the spermogram also provides information revealing a pathology of vas deferens. This is the quantitative and qualitative analysis of spermatozoids. Depending on the clinical status, an azoospermia, hypospermia or decreased spermatozoid vitality can be seen on the spermogram. ${ }^{3}$ However hypospermia (sperm volume $<1.5 \mathrm{ml}$ ), acidic $\mathrm{pH}$ are pathognomonic biological sign ${ }^{4}$ of BAVD associated with absence of Seminal Vesicle.

An obstructive azoospermia is found in subjects with cystic fibrosis or bilateral congenital absence of vas deferens. ${ }^{5}$ This situation explains the fertility disorders in the men concerned. It follows that the CFTR gene is at the centre of male fertility. ${ }^{6}$ The manifestation of cystic fibrosis and the risk of CBAVD are genetically linked. Mutant alleles of CFTR exist in men with congenital absence of vas deferens. cystic fibrosis is highly heterogenic mutations. ${ }^{7-9}$

Magnetic resonnance imaging is the most appropriate for diagnosing the absence of vas deferens and seminal vesicles. This examination makes it possible to visualize the different pelvic anatomical elements it gives better precision than the endorectal ultrasound of the prostate, vas deferens and seminal vesicles. ${ }^{10}$

We report the case of our patient to find out the diagnostic and therapeutic difficulties related to this rare cause of infertility.

\section{Case presentation}

A 32-year-old patient. Obesity surgery operated at age 25 by Bypass with loss of about $30 \mathrm{kgs}$, type 2 diabetes. Married for 5 years, he had regular and normal sexual intercourses with his 26-yearold companion who had a normal health check-up. Clinically there were no signs of hypogonadism, no urinary symptoms, no erectile dysfunction. The digital rectal examination did not record any abnormalities. Transrectal ultrasound revealed agenesis of a left seminal vesicle, hypotrophy of the right seminal vesicle, without individualization of vas deferens. Pelvic MRI noted an absence of bilateral vas deferens and agenesis of a left seminal vesicle and hypotrophy of the right seminal vesicle (Figure 1). Semen analyses were carried out and Absence of spermatozoa confirmed: volume $1 \mathrm{ml}$, and $\mathrm{pH}$ of semen 7,8 sperm concentration $0 \mathrm{M} / \mathrm{ml}$, vitality $0 \%$ and motility $0 \%$ (azoospermia).Hormonal assessment, FSH and inhibine were normal, normal testosterone. Constitutional karyotyping and search for microdeletions in the AZF regions of the $\mathrm{Y}$ chromosome were realized and noted no microdeletion in the AZF gene but the presence of an ACFTR mutation. Following these explorations we performed a microsurgical epididymal sperm aspiration (MESA) for an intracytoplasmic injection that was successfully carried out and allowed a paternity after the first attempt. 

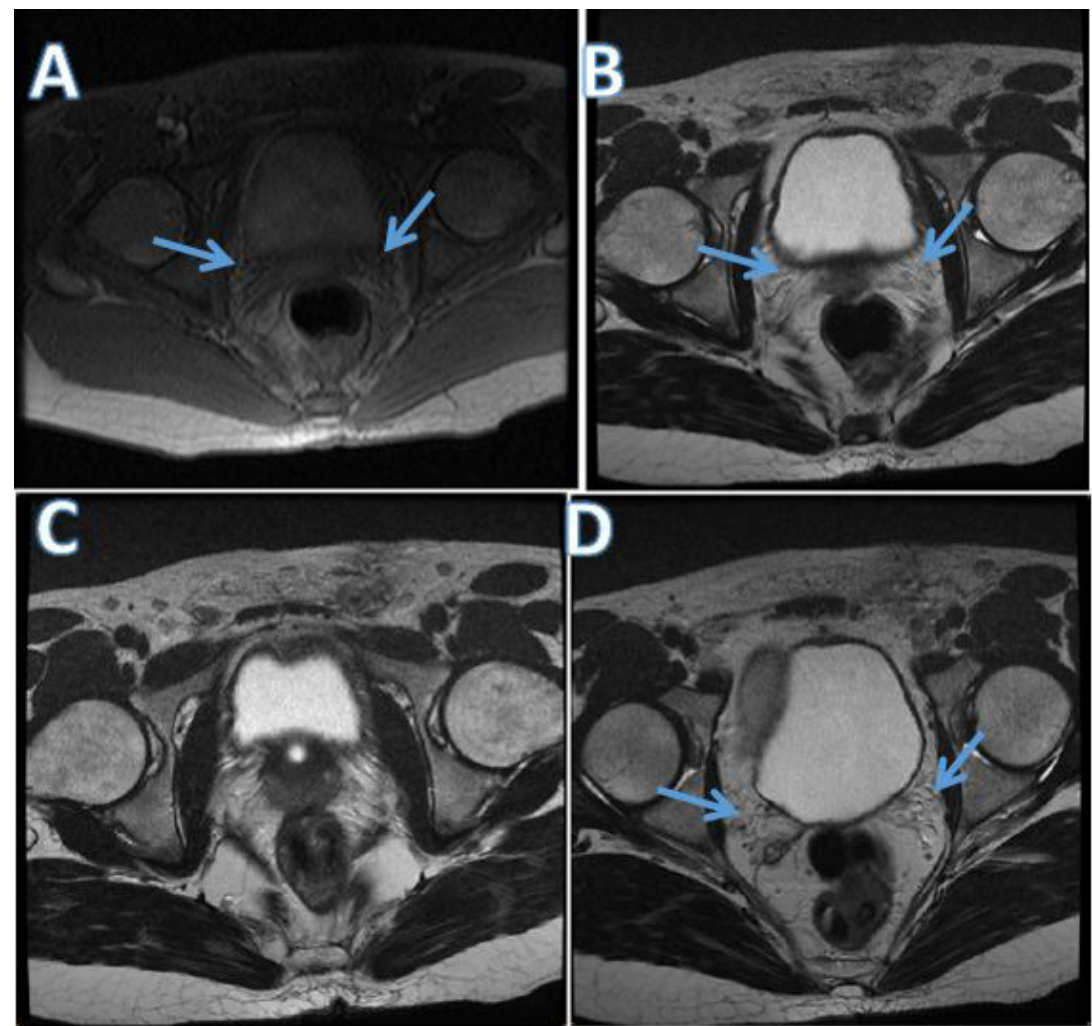

Figure I Pelvic MRI A, B, C noted an absence of bilateral vas deferens (CBAVD).

$\mathrm{D}$ agenesis of a left seminal vesicle and hypotrophy of the right seminal vesicle.

\section{Discussion}

Endorectal imaging of the prostate by ultrasound allows visualization of the seminal vesicles and vas deferens. It is indicated in case of clinical suspicion of absence of vas deferens in infertility consultations in men. ${ }^{11}$ Indeed endorectal ultrasound of the prostate is very accessible to the urologist to detect absence of vas deferens in male consulting for primary infertility.

Magnetic resonance imaging is the reference imaging for the description of pelvic anatomy. As part of the diagnosis of bilateral agenesis of vas deferens it allows a marked visualization of the absence of vas deferens and seminal vesicles. ${ }^{12}$

Fertility in patients with cystic fibrosis has been demonstrated as well as the fact that these same patients are also affected by CBAVD. ${ }^{13}$ The spermogram is the main laboratory test in the fertility check. In the case of patients without vas deferens, it is possible to diagnose an obstructive azoospermia. ${ }^{14}$ In our case, our patient had an absence of bilateral vas deferens on the MRI performed. His karyotype did not record microdeletion of the AZDF gene but the presence of an ACFTR mutation.

According to a comparative study on cystic fibrosis and congenital absence of vas deferens, it appears that they have in common the mutation of the CFTR gene. It is recommended that all men with CBAVD be tested for CFTR gene, so it is important that they receive genetic advice on the disease. Men who have a congenital absence of vas deferens do indeed have a good spermatogenesis, however it is necessary for the latter to resort to the techniques of microsurgery such as microepididymal sperm aspiration (MESA), percutaneous epididymal sperm aspiration (PESA), or testicular sperm extraction (TESE) as part of the medical assistance to procreation in order to become fertile. Other studies have also shown that the fertility success rate in patients with congenital absence of vas deferens was low in medical assistance to procreation compared to other patients also candidates for these techniques. ${ }^{15,16}$ Recent assisted human reproduction techniques like Intracytoplasmic Sperm Injection (ICSI) or In Vitro Fertilization (FIV) offer very good results. ${ }^{17}$

Assisted human reproduction techniques produce positive results in the case of couples whose partner carries a bilateral absence of vas deferens. Several procedures are therefore used to extract and keep sperm in good conditions to make them fertile. Current techniques are based on the micro-surgery of suction of spermatozoids at the level of the testicular epididymis. ${ }^{18}$

\section{Conclusion}

The lack of deferens palpation or atretic cord clinically with spermatic hypovolemia on the spermogram remain signs of CAVD. The search for mutations of the CFTR gene and endorectal ultrasound and pelvic MRI are essential in the assessment of an obstructive azoospermia to deferens(s) not palpated(s). Deferens agenesis is considered a minor form of cystic fibrosis. A Genetic Council is necessary to search for other associated genetic pathologies. Surgical sperm collection is the hope for obtaining a pregnancy in the partner for this type of patients.

\section{Conflicts of interest}

The author declares no conflict of interest.

\section{Acknowledgments}

None. 


\section{Funding}

None.

\section{References}

1. Lin $\mathrm{C}-\mathrm{H}$, Huang $\mathrm{T}-\mathrm{Y}$. Congenital bilateral absence of the vas deferens (CBAVD) with bilaterally present seminal vesicles. Urol Case Rep. 2020;31:101131.

2. Li C-Y, Jiang L-Y, Chen $\mathrm{W}-\mathrm{Y}$, et al. CFTR is essential for sperm fertilizing capacity and is correlated with sperm quality in humans. Hum Reprod. 2010;25(2):317-327.

3. Ferlin A, Stuppia L. Diagnostics of CFTR-negative patients with congenital bilateral absence of vas deferens: which mutations are of most interest? Expert Rev Mol Diagn. 2020;20(3):265-267.

4. Roger Mieusset, Eric Bieth, Myriam Daudin, et al. Male partners of infertile couples with congenital unilateral absence of the vas deferens are mainly non-azoospermic - Mieusset - 2020 - Andrology. 2020;8(3):645653.

5. Van Mourik P, van Haaren P, Kruisselbrink E, et al. R117H-CFTR function and response to $\mathrm{VX}-770$ correlate with mRNA and protein expression in intestinal organoids. J Cyst Fibros Off J Eur Cyst Fibros Soc. 2020;19(5):728-732.

6. Santi CD, Fernández EF, Gaul R, et al. Precise Targeting of miRNA Sites Restores CFTR Activity in CF Bronchial Epithelial Cells. Mol Ther. 2020;28(4):1190-1199.

7. Ferec C, Cutting GR. Assessing the Disease-Liability of Mutations in CFTR. Cold Spring Harb Perspect Med. 2012 1;2(12):a009480.

8. Tamburino L, Guglielmino A, Venti E, et al. Molecular analysis of mutations and polymorphisms in the CFTR gene in male infertility. Reprod Biomed Online. 2008;17(1):27-35.
9. Patel B, Parets S, Akana M, et al. Comprehensive genetic testing for female and male infertility using next-generation sequencing. $J$ Assist Reprod Genet. 2018;35(8):1489-1496.

10. Chiang $\mathrm{H}-\mathrm{S}$, Lin $\mathrm{Y}-\mathrm{H}, \mathrm{Wu} \mathrm{Y}-\mathrm{N}$, et al. Advantages of magnetic resonance imaging (MRI) of the seminal vesicles and intra-abdominal vas deferens in patients with congenital absence of the vas deferens. Urology. 2013;82(2):345-351.

11. Schlegel PN, Shin D, Goldstein M. Urogenital anomalies in men with congenital absence of the vas deferens. J Urol. 1996;155(5):1644-1648.

12. Arora SS, Breiman RS, Webb EM, et al. CT and MRI of Congenital Anomalies of the Seminal Vesicles. Am J Roentgenol. 2007;189(1):130 135.

13. Male Infertility: Understanding, Causes and Treatment | RAJENDER SINGH | Springer.

14. EAU-Guidelines-Male-Infertility. 2016.

15. Silber SJ, Balmaceda J, Borrero C, et al. Pregnancy with sperm aspiration from the proximal head of the epididymis: a new treatment for congenital absence of the vas deferens. Fertil Steril. 1988;50(3):525-528.

16. Nicopoullos JDM, Gilling-Smith C, Almeida PA, Ramsay JWA. The results of 154 ICSI cycles using surgically retrieved sperm from azoospermic men. Hum Reprod Oxf Engl. 2004;19(3):579-585.

17. Bouyé S, Marcelli F, Ghoneim T, et al. Description andrologique d'une population azoosperme avec une agénésie des canaux déférents consultant pour infertilité. Prog En Urol. 2014;24(2):132-137.

18. Wong R, Gu K, Ko Y, Patel P. Congenital absence of the vas deferens: Cystic fibrosis transmembrane regulatory gene mutations. Best Pract Res Clin Endocrinol Metab. 2020;34(6):101476. 